\title{
Índices de sorción de metales pesados en suelos urbanos: el caso de Morelia, Michoacán
}

\author{
Francisco Bautista, Elsy Campuzano, Carmen Delgado, Avto Goguitchaichvili
}

\begin{abstract}
Francisco Bautista Elsy Campuzano

leptosol@ciga.unam.mx

Laboratorio Universitario de Geofisica Ambiental, Centro de Investigaciones en Geografía Ambiental, Universidad Nacional Autónoma de México. Antigua Carretera a Pátzcuaro No. 8701, Col. Ex-Hacienda de San José de la Huerta. C.P. 58190. Morelia, Michoacán, México.
\end{abstract}

\section{Elsy Campuzano}

Universidad Politécnica del Estado de Guerrero. Carretera Federal Iguala-Taxco, Km 105. C.P. 40321. Taxco de Alarcón, Guerrero, México.

\section{Garmen Delgado}

Universidad Michoacana de San Nicolás de Hidalgo, Instituto de Investigaciones en Ciencias de la Tierra (INICIT-UMSNH). Avenida universidad No. 1471, Giudad Universitaria. C.P. 58066. Morelia, Michoacán, México.

\section{Avto Goguitchaichvili}

Laboratorio Universitario de Geofisica Ambiental, Instituto de Geofísica, Unidad Michoacán. Universidad Nacional Autónoma de México. Antigua Carretera a Pátzcuaro No. 8701, Gol. Ex-Hacienda de San José de la Huerta. C.P. 58190. Morelia, Michoacán, México.

BOL. SOC. GEOL. MEX. 2017

VOL. 69 NO. 2

P. $433-445$

Manuscrito recibido: Agosto 10, 2016 Manuscrito corregido: Febrero 7, 2017 Manuscrito aceptado: Febrero 15, 2017

\section{RESUMEN}

Debido al acelerado crecimiento urbano y al desarrollo de las actividades antrópicas, como las de giro industrial, flujo vehicular y generación de residuos sólidos urbanos, se han liberado grandes cantidades de metales pesados al medio ambiente, contribuyendo así a la contaminación de los suelos urbanos. Esta contaminación ha ido en aumento y se ha convertido en objeto de estudio, sin embargo, la capacidad del suelo para adsorber metales pesados es poco estudiada en la actualidad. El objetivo de este trabajo fue evaluar y/o desarrollar tres índices de sorción de metales pesados, así como su validación matemática mediante árboles de decisión para clasificación. El estudio se realizó en la zona urbana de la ciudad de Morelia, Michoacán, donde se tomaron de manera sistemática 100 muestras de suelo urbano superficial. Posteriormente, se determinó el contenido de materia orgánica, $\mathrm{pH}$, capacidad de intercambio catiónico, porcentaje de arcillas, estructura, pedregosidad y densidad aparente. El índice de Lehmann fue evaluado y se desarrollaron dos nuevos índices: el índice Lehmann modificado y el índice Bautista-Campuzano. La validación matemática de los tres índices se realizó mediante la elaboración de árboles de decisión para clasificación con el software WEKA. En el índice Lehmann se consideró principalmente el porcentaje de arcillas, estructura y pedregosidad; el índice Lehmann modificado utilizó la capacidad de intercambio catiónico, materia orgánica, pedregosidad y pH; y finalmente, el índice Bautista-Campuzano consideró de mayor importancia al $\mathrm{pH}$, materia orgánica, arcillas, pedregosidad y densidad aparente. Los tres índices mostraron congruencia entre las propiedades del suelo y las clases de sorción de metales pesados con un coeficiente Kappa $>0.7$. No obstante, el índice Bautista-Campuzano es el que mostró mayor congruencia teórica según el árbol de decisión para clasificación. Este estudio revela la importancia de la evaluación matemática y teórica de los índices mediante los árboles de decisión para clasificación. Por tal motivo, se considera que el índice Bautista-Campuzano es la metodología más eficiente para la generación de información y la toma de decisiones relacionada con el mejoramiento de los suelos en zonas urbanas para aumentar la sorción de metales pesados.

Palabras clave: contaminación; ciudad; suelos superficiales; árboles de decisión para clasificación.

\section{ABSTRACT}

Due to rapid urban growth and significant increase of human activities such as industrialization, use of motor vehicles and the generation of urban solid waste a large amounts of heavy metals are systematically released into the environment contributing to the pollution of urban soils. However, the ability of soil to adsorb heavy metals is still poorly studied. The aim of this investigation was the evaluation and / or development of three heavy metal sorption indices and their mathematical validation using decision trees for classification purposes. The sampling was carried out within the urban area of the city of Morelia, State of Michoacan, Mexico. In total 100 samples of topsoil were collected and the content of organic matter, $p H$, cation exchange capacity, percentage of clay, structure, stoniness and bulk density were analyzed. The Lehmann index was evaluated and two new indices were developed, the modified Lehmann index and the Bautista-Campuzano index. The mathematical validation and development of decision trees were performed using the WEKA software. The Lehmann index took into account mainly the percentage of clays, aggregates and stoniness. The modified Lehmann index used the cation exchange capacity, organic matter, stoniness and pH. Finally the Bautista-Campuzano index considered the $p H$, organic matter, clays, stoniness and bulk density. The three indices showed congruence between soil properties and sorption classes of heavy metals with a Kappa coefficient greater than 0.7. However, the Bautista-Campuzano index showed greater theoretical consistency according to the decision tree for classification purpose. This study reveals the importance of the mathematical and theoretical evaluation of the indices using decision trees for classification. For this reason, we consider that the Bautista-Campuzano index is the most efficient methodology for generating information and decision-making related to the improvement of soils in urban areas to increase the sorption of heavy metals.

Keyzords: Pollution, Cities, Top soils, Decision trees for classification. 


\section{Introducción}

Estudios realizados en suelos urbanos en varias ciudades alrededor del mundo mostraron altas concentraciones de metales pesados (MP), tales como: Cd, Cr, Cu, Pb, Ni, V y Zn (Lu y Bai, 2010; Cejudo-Ruíz et al., 2015; Cortés et al., 2015; Ihl et al., 2015; Liu et al., 2016). Los metales pesados en ambientes urbanos pueden ser ingeridos y/o inhalados y llegar a constituirse en un problema de salud ya que muchos de ellos ocasionan enfermedades graves en humanos. No obstante, son muy escasos los sistemas de monitoreo debido a que los diagnósticos mediante los tradicionales análisis químicos requieren tiempo, dinero y esfuerzo, lo que impide su realización. Por esta razón, se han realizado muchos esfuerzos para encontrar tecnologías rápidas y económicas denominadas proxy, entre las que destaca el magnetismo edáfico (Aguilar-Reyes et al., 2012, 2013a, 2013b; Cejudo-Ruíz et al., 2015; Sánchez-Duque et al., 2015) y recientemente el color del suelo (Cortés et al., 2015). Sin embargo, existen pocos estudios sobre la evaluación de la capacidad del suelo para la sorción de los metales pesados con técnicas rápidas y de bajo costo, diferentes a las técnicas empíricas de isotermas de sorción química (Bradl, 2004).

Teóricamente se sabe que las propiedades que participan en la sorción de MP en los suelos pueden clasificarse en primarias y secundarias. En el grupo de las propiedades primarias se encuentra en orden de importancia: el $\mathrm{pH}$ como propiedad más influyente ya que de éste depende la movilidad de los metales pesados (Davis, 2000), materia orgánica $(\mathrm{MO})$ por su capacidad quelatante (Alloway y Trevors, 2013), arcillas por su capacidad de sorción de cationes (Towhid, 2013) y, la agregación debido a la estabilidad física que le da al suelo (Bronick y Lal, 2005). En el grupo de las propiedades secundarias se encuentran la cantidad de tierra fina calculada con la densidad aparente, pedregosidad y espesor del horizonte (Jordán, 2006). La idea es que se identifiquen las propiedades clave en la sorción de los metales pesados en el suelo para: a) identificar las zonas de menor capacidad de amortiguamiento a la contaminación por metales pesados; y b) diseñar formas de recuperación de suelos contaminados.

La ciudad de Morelia tiene una superficie de 85 $\mathrm{km}^{2}$, con una población de 597511 habitantes por lo que es considerada como una ciudad media en crecimiento; además, su área metropolitana consta de 729279 habitantes y existen 332381 mil vehículos automotores registrados en circulación (INEGI, 2014). Las principales actividades económicas del municipio de Morelia son: comercio, turismo y servicios $(63.7 \%$ ); industria de la construcción y manufacturera (25.9\%); y la agricultura $(10.4 \%)$ (INEGI, 2010). El sector industrial incorpora los siguientes giros: elaboración de aceite comestible, productos químicos, resinas, harina, fundición, industria del plástico, calderas, dulces en conservas, embotellamiento de agua y refrescos, fabricación de generadores eléctricos, turbinas hidráulicas y de vapor y productos de celulosa y papel (INECG-SEMARNAT, 2010). Por lo anterior, se considera que la mayor influencia en cuanto a la generación de partículas con metales pesados proviene, principalmente, del tráfico vehicular (Aguilar et al., 2012).

El objetivo de este estudio consistió en la evaluación de tres índices de sorción de metales pesados en suelos urbanos utilizando como estudio de caso la ciudad de Morelia, Michoacán, México.

\section{Material y métodos}

\subsection{ZONA DE ESTUDIO}

La ciudad de Morelia está localizada1946’06” LN y $101^{\circ} 11^{\prime} 22^{\prime \prime}$ LW al centro-norte del estado de Michoacán, a una altitud de 1920 msnm (Figura 1).

Presenta un clima subhúmedo con temperatura media anual de $17.5^{\circ} \mathrm{C}$ y precipitación pluvial de $773 \mathrm{~mm}$. Los vientos dominantes proceden del suroeste y noreste y son variables en julio y agosto con intensidades de 2.0 a $14.5 \mathrm{~km} \mathrm{~h}^{-1}$. El basamento está conformado por rocas ignimbríticas 
bien cementadas. Los grupos principales de suelos son: Vertisoles, Luvisoles, Acrisoles, Leptosoles y Andosoles.

\subsection{DISEÑO DEL MUESTREO}

Se diseñó un muestreo sistemático sobre la traza urbana, con 100 puntos de muestreo a una distancia del km (Figura 1). El muestreo se realizó durante la época de sequía debido a que según Bautista et al. (2011) la presencia de lluvia puede modificar las condiciones y propiedades del suelo que se deseen analizar. La muestra de suelo se extrajo con un cilindro de PVC (policloruro de vinilo) de $5 \mathrm{~cm}$ de largo por $5 \mathrm{~cm}$ de diámetro interno. Previo a la introducción del cilindro se retiró la hojarasca, las piedras en la superficie o cualquier otro material (Delgado et al., 2015). El suelo se depositó en una bolsa de plástico previamente etiquetada.

Las muestras se secaron a la sombra y a temperatura ambiente durante dos semanas, se molieron en un mortero y con pistilo de madera, y se tamizaron con una malla del número 10 con luz de 2 $\mathrm{mm}$.

\subsection{ANÁLISIS FÍSICOS Y QUÍMICOS}

Las propiedades físicas y químicas de los suelos estudiados se realizaron con los siguientes procedimientos: $\mathrm{pH}$ de una mezcla suelo-agua con una relación 1:2, se determinó el porcentaje de carbono orgánico (CO) (Nelson y Sommers, 1982); capacidad de intercambio catiónico (CIC) con el método de acetato de amonio (Rhoades, 1982); y el porcentaje de arcillas con el método de Bouyoucos (Okalebo et al., 1993). En el análisis de la estructura se consideró: forma, tamaño y estabilidad de los agregados; mientras que la densidad aparente se midió con la técnica del cilindro $(\mathrm{FAO}$, 2006). Finalmente, la pedregosidad se determinó mediante la diferencia entre el peso de la muestra sin tamizar y el de la muestra tamizada. Los resul-

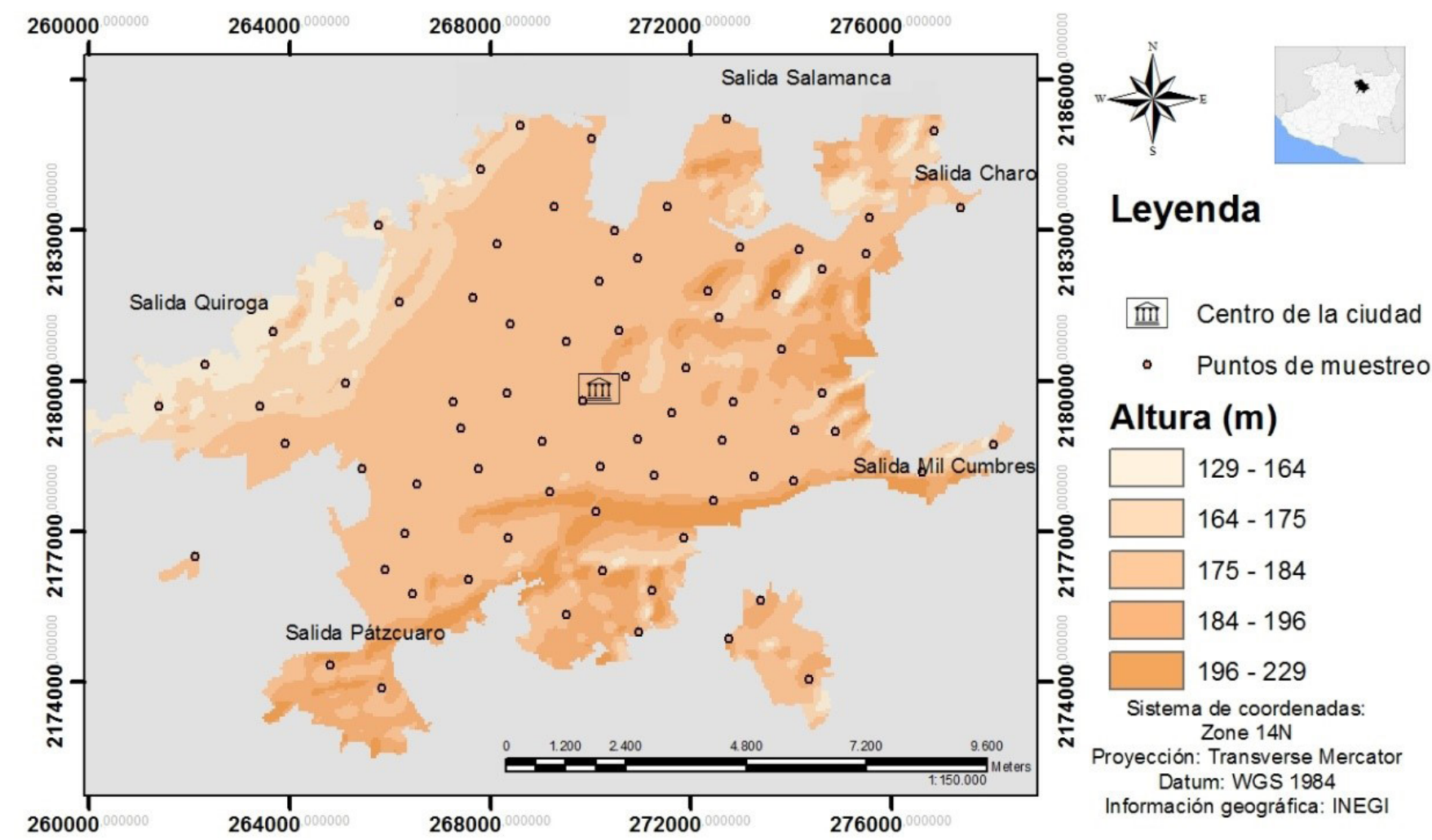


tados se expresaron en porcentajes.

\subsection{EVALUAGIÓN DE LOS ÍNDIGES}

Tres índices de sorción de metales pesados se trabajaron utilizando el horizonte superficial de los suelos urbanos de la ciudad de Morelia: 1) índice Lehmann (Lehmann et al., 2013); y dos nuevos índices; 2) índice Lehmann modificado; y 3) índice Bautista-Campuzano.

En el índice Lehmann las ecuaciones utilizadas, así como los valores dados al $\mathrm{pH}$ y a la estructura predominante (tipo de agregado) (Tabla 1) se tomaron del manual Técnica de evaluación y categorización del suelo (TUSEC, por sus siglas en inglés) (Lehmann et al., 2013; Bautista et al., 2015). Cabe mencionar que dichas ecuaciones fueron modificadas debido a que están diseñadas para evaluar perfiles completos de suelo. El procedimiento fue el siguiente: primero se obtuvo el porcentaje de arcilla, posteriormente se transformó a fracción y se multiplicó según el tipo de agregado con la siguiente ecuación:

$$
C L Y=\left(\frac{A m}{100}\right) * A G G \quad(\text { Ecuación 1) }
$$

Donde, CLY es el valor de arcilla, Am corresponde al porcentaje de arcillas y $A G G$ son los valores de agregación del suelo.

Posteriormente,se calculó el factor arcilla mediante la siguiente ecuación:

Tabla 1. Factor pH y de agregación según Lehmann et al. (2013).

\begin{tabular}{|c|c|l|c|}
\hline $\mathbf{p H}\left(\mathbf{C a C l}_{2}\right)$ & Factor $\mathbf{p H}$ & $\begin{array}{l}\text { Estructura } \\
\text { predominante en el } \\
\text { suelo }\end{array}$ & $\begin{array}{l}\text { Valor dado a la } \\
\text { estructura } \\
\text { predominante del } \\
\text { suelo }\end{array}$ \\
\hline$\geq 6.5$ & 1 & $\begin{array}{l}\text { Bloques angulares. } \\
\text { Columnar, prismática }\end{array}$ & 0.5 \\
\hline $5.5<6.5$ & 0.8 & $\begin{array}{l}\text { Columinar. } \\
\text { o lames }\end{array}$ \\
\hline $5.0<5.5$ & 0.5 & $\begin{array}{l}\text { Otros (Bloques } \\
\text { subangular, granular o } \\
\text { migajosa) }\end{array}$ & \multicolumn{1}{|c|}{1} \\
\hline $4.0<5.0$ & 0.3 & \\
\hline$<4$ & 0.1 & \\
\hline
\end{tabular}

$$
C L Y F=T H *\left(\frac{1-C R}{100}\right) *(C L Y) *(p H F) \quad(\text { Ecuación 2) }
$$

Donde, CLYF corresponde al Factor arcillas, TH es el espesor del horizonte, $C R$ es el porcentaje de pedregosidad, CLY es el porcentaje de arcilla, y $p H F$ es el factor $\mathrm{pH}$ de la muestra.

Después, la materia orgánica se transformó a fracción ( $H U$ o fracción humus) de la siguiente manera:

$$
H U=\frac{\% H U m}{100}
$$

(Ecuación 3)

Donde, HU es la fracción humus, \%HUm es el porcentaje de $\mathrm{MO}$ en la muestra.

$\mathrm{El}$ factor humus $(H U F)$ se calculó multiplicando la fracción humus por el factor $\mathrm{pH}$ y se convirtió el valor en unidades por superficie.

$$
H U F=T H * 10 *\left(\frac{1-C R}{100}\right) * H U * p H F \quad(\text { Ecuación 4) }
$$

Los valores de los factores arcillas y humus se agruparon en cinco clases de acuerdo con los valores de sorción: Clase 1 muy alta; Clase 2 alta; Clase 3 media, Clase 4 baja; y Clase 5 muy baja. Los grados de sorción de los factores humus y arcillas se compararon entre sí y se escogió como indicador al que tuvo la mayor sorción.

El cálculo del índice Lehmann modificado es similar al anterior ya que la ecuación utilizada es la misma; sin embargo, en este índice se substituyó al porcentaje de arcillas por la CIC, ya que esta propiedad se consideró un mejor indicador de la sorción metales pesados en el suelo debido a que es un indicador inmediato de la disponibilidad que tiene el suelo para intercambiar cationes (Porta et al., 2003).

$$
C I C F=T H *\left(\frac{1-C R}{100}\right) * C I C * p H F \quad(\text { Ecuación 5) }
$$

Donde, CICF es el factor CIC por muestra, TH es el espesor del horizonte, $C R$ es el porcentaje de pedregosidad, $C I C$ es la capacidad de intercambio de cationes y el $p H F$ es el factor $\mathrm{pH}$ de la muestra. 
En el índice de sorción "Bautista-Campuzano" (IABC), los porcentajes de arcillas y carbono orgánico $(\mathrm{CO})$ se transformaron a unidades $\mathrm{de} \mathrm{kg} / \mathrm{m}^{2}$ con ayuda de la aplicación SOC+ (Bautista et al., 2016). Esta aplicación fue diseñada para teléfonos inteligentes, y permite estimar el carbono orgánico en unidades de peso por superficie considerando la profundidad, densidad aparente y pedregosidad (Bautista et al., 2016). Este índice le da mayor importancia al contenido de tierra fina calculada con la pedregosidad, densidad aparente y espesor del horizonte. El índice de sorción se calculó de la siguiente manera:

$$
I A B C=\frac{p H F+A m F+C O F}{3} \quad \text { (Ecuación 6) }
$$

Donde, IABC corresponde al índice de sorción Bautista-Campuzano; $p H F$ es el factor $\mathrm{pH}$, el cual se determinó a partir de los valores medidos de $\mathrm{pH} ; A m F$ es el factor arcillas y $C O F$ es el factor carbono orgánico. Éstos fueron obtenidos con la aplicación SOC+. Finalmente, con los valores obtenidos se formaron cinco clases para cada factor: clase 5 muy alta, 4 alta, 3 media, 2 baja y 1 muy baja (Tabla 2).

\subsection{VALIDACIÓN DE LOS ÍNDICES UTILIZANDO ÁRBOLES DE DEGISIÓN}

Se construyó una base de datos en Excel con las propiedades físicas y químicas, y con el valor de los índices desarrollados, después se transformó en archivo .csv. Este formato proviene del inglés com-

Tabla 2. Valores de los factores según cada propiedad del suelo para la obtención del índice Bautista-Campuzano.

\begin{tabular}{|c|c|c|c|}
\hline $\mathbf{p H}$ & Arcillas en $\mathbf{~ k g} / \mathbf{m}^{2}$ & $\mathbf{C O}$ en $\mathbf{~ k g} / \mathbf{m}^{2}$ & Factor \\
\hline$>7$ & $3-3.5$ & $>0.8$ & 5 \\
\hline $6.5-7$ & $2.5-3$ & $0.6-0.8$ & 4 \\
\hline $6-6.5$ & $2-2.5$ & $0.4-0.6$ & 3 \\
\hline $5.5-6$ & $1.5-2$ & $0.2-0.4$ & 2 \\
\hline $5-5.5$ & $<1.5$ & $<0.2$ & 1 \\
\hline
\end{tabular}

ma-separated values, el cual se utiliza para representar datos en forma de tabla en donde las columnas se separan por comas. Posteriormente los datos se analizaron mediante minería de datos con el software WEKA (Legrand y Nicoloyannis, 2005; Hall et al., 2009), mediante la siguiente secuencia: 1) limpieza de datos, y 2) aplicación del algoritmo "J48"con la opción Use training set para la elaboración de los árboles de decisión.

El índice de sorción se seleccionó al comparar los árboles de decisión, la teoría conocida sobre la sorción de MP en el suelo y el coeficiente Kappa, el cual refleja las concordancias observadas sobre el total de observaciones hechas (Cerda y Villarroel, 2008).

\subsection{ANÁLISIS ESPACIAL}

La base de datos se realizó con los números de muestra, las coordenadas geográficas y los valores de los índices de sorción de metales pesados. El análisis espacial se llevó a cabo con el programa GS+ (Robertson, 2008) donde se efectúan los estudios de estadística descriptiva; posteriormente se llevó a cabo un análisis geoestadístico mediante la autocorrelación espacial y el diseño de los semivariogramas con la siguiente ecuación:

$$
\gamma(h)=\frac{1}{2 n} \sum_{i=1}^{n}\left[Z\left(x_{i}\right)-Z\left(x_{i}+h\right)\right]^{2}(\text { Ecuación } 7)
$$

Donde: $\boldsymbol{\gamma}(h)$ denota el valor del semivariograma experimental para todos los pares de datos de los índices de sorción a una distancia $\mathrm{h} ; Z(X)$ muestra los valores de los índices en un sitio $X ; Z z\left(X_{i}+\right.$ $h$ ) e indica los valores de los índices en diferentes puntos, los cuales son separados de $X_{i}$ en distancia discreta $\mathrm{h} ; X_{i}$ expresa el sitio georeferenciado y el valor de los índices de contaminación; $n$ indica los números de pares separados a la distancia $h$ (Hernández-Stefanoni y Ponce-Hernández, 2006; Delgado et al., 2010; Zhang et al., 2014; Cortés et al., 2015).

Se obtuvo el modelo que mejor se ajustó al semivariograma experimental, y por último se realizaron los diagramas de superficie continua con el método de interpolación kriging ordinario $(\mathrm{KO})$. 
El mapa fue elaborado con el software ArcGIS 10.2 (ESRI, 2004) con un datum de referencia horizontal y el Sistema Geodésico Mundial 84 (WGS84).

\section{Resultados y Discusión}

\subsection{APLIGACIÓN DE LOS ÍNDIGES}

Con la aplicación del índice Lehmann se lograron valores bajos de sorción comparados con los valores establecidos para evaluación de perfiles completos (Lehmann et al., 2013); sin embargo, en este caso sólo se aplicó a los suelos superficiales (5 $\mathrm{cm}$ de profundidad). Los suelos estudiados mostraron más valores bajos en el factor humus que en el factor arcillas (Tabla 3). Con el índice Lehmann se obtuvo el mayor número de casos con las clases alta y muy alta en comparación con los índices Lehmann modificado y Bautista-Campuzano (Figura 2).

Con el índice Lehmann modificado la CIC tomó un papel relevante. Esta propiedad del suelo fue la que hizo la diferencia entre las clases de sorción de metales pesados (Tabla 4) debido a que los valores de la CIC se encontraron en un intervalo amplio que va desde $7 \mathrm{cmol}(+) \mathrm{kg}^{-1}$ hasta $70 \mathrm{cmol}(+) \mathrm{kg}^{-1}$. La diversidad de texturas, contenido y tipo de coloides y cantidad de materia son la consecuencia de la diversidad de suelos y rocas (Areygue-Rocha et al., 2002) que se encuentran en la ciudad de

Tabla 3. Clases de sorción para los factores arcilla y humus en el índice Lehmann.

\begin{tabular}{|l|l|l|}
\hline Factor arcillas & \multicolumn{1}{|c|}{ Factor Humus } & Clases de sorción \\
\hline$>0.20$ & $>0.12$ & $1=$ Muy alta \\
\hline$>0.15-0.20$ & $>0.09-0.12$ & $2=$ Alta \\
\hline$>0.10-0.15$ & $>0.06-0.09$ & $3=$ Media \\
\hline$>0.05-0.10$ & $>0.03-0.06$ & $4=$ Baja \\
\hline$<0.05$ & $<0.03$ & $5=$ Muy baja \\
\hline
\end{tabular}

Morelia

El índice Lehmann modificado generó más casos en las clases media y baja (Figura 2) en comparación con el índice de Lehmann. Con ambos índices se obtuvieron casos en todas las clases de sorción de metales pesados.

El índice Bautista-Campuzano es un índice de mayor facilidad y manejo debido a: 1) las propiedades utilizadas en este índice son convertidas a factores antes de ser introducidas en la ecuación; 2) los factores considerados tienen los mismos valores que las clases de sorción (Tabla 5). Además, la ecuación del índice Bautista-Campuzano se compone del valor de los factores derivados de las propiedades del suelo; caso contrario son las ecuaciones de los índices Lehmann y en menor magnitud del índice Lehmann modificado (Lehmann et al., 2013). Esta situación facilita el entendimiento y aplicación del índice.

Con el índice Bautista-Campuzano se disminuyeron los casos en la clase muy baja y no se presentó ningún caso en la clase muy alta. Por el contrario, la clase media de sorción de metales pesados presentó más casos que los otros dos índices aplicados (Figura 2). Esta situación se presentó como consecuencia de que el índice Bautista-Campuzano tomó en consideración la cantidad de tierra fina como factor importante debido que es allí y no en la fracción gruesa donde se da la sorción de los metales pesados (Lehmann y Stahr, 2007).

\subsection{VALIDAGIÓN DE LOS ÍNDIGES GON ÁRBOLES DE DEGISIÓN PARA GLASIFICACIÓN}

Los árboles de decisión modelan y revelan el proceso de clasificación ya que el uso de las ecuaciones sólo dan el resultado numérico lo cual impide conocer el peso de cada propiedad que se considera en la aplicación del índice (Aggarwal, 2015). El índice Lehmann tomó en cuenta tres propiedades para modelar el proceso de clasificación: el porcentaje de arcillas, tipos de agregados (en algunos casos el modelo Lehmann determina en este punto el grado de sorción) y pedregosidad del suelo (Figura 3). 


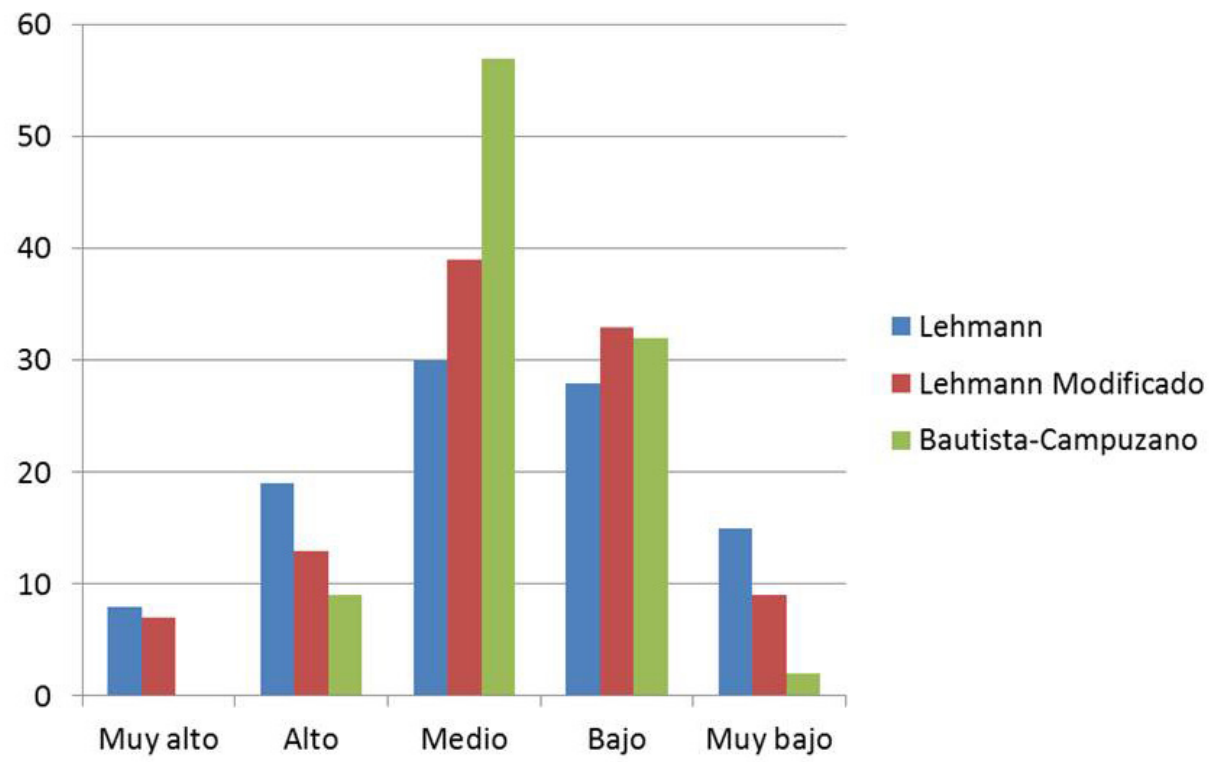

Figura 2 Comparación entre las clases de sorción de metales pesados utilizando los índices.

Tabla 4. Clases de sorción del ìndice Lehmann modificado.

\begin{tabular}{|c|l|}
\hline Factor CIC & \multicolumn{1}{|c|}{ Clases de sorción } \\
\hline $40-50$ & $1=$ Muy alto \\
\hline $30-40$ & $2=$ Alto \\
\hline $20-30$ & $3=$ Medio \\
\hline $10-20$ & $4=$ Bajo \\
\hline$<10$ & $5=$ Muy bajo \\
\hline
\end{tabular}

En el árbol de decisión para el índice Lehmann modificado en primer lugar se tomó en cuenta a la CIC, después a la materia orgánica y se reconsideró a la CIC (se comienzan a definir las clases de sorción). Después se consideraron al pH y a la pedregosidad los cuales son los factores finales para la determinación del grado de sorción (Figura 4). A partir de este árbol de decisión se observa que las propiedades consideradas para hacer la clasificación aumentan a cuatro en comparación con las tres consideradas en el índice Lehmann. El proceso de clasificación se mejoró debido a que la CIC es una propiedad de relevancia en la sorción de metales pesados (Bradl, 2004).

El árbol de decisión del índice Bautista-Campu-
Tabla 5. Clases de sorción, ìndice de sorción Bautista-Campuzano (IABC).

\begin{tabular}{|c|l|}
\hline Factor B-C & \multicolumn{1}{|c|}{ Clases de sorción } \\
\hline 5 & $5=$ Muy alto \\
\hline 4 & $4=$ Alto \\
\hline 3 & $3=$ Medio \\
\hline 2 & $2=$ Bajo \\
\hline 1 & $1=$ Muy bajo \\
\hline
\end{tabular}

zano tomó en cuenta cinco propiedades del suelo: $\mathrm{pH}$, pedregosidad y carbono orgánico, para después retomar al $\mathrm{pH}$, pedregosidad e incluir a las arcillas y finalmente tomó en cuenta a la densidad aparente (Figura 5). En este caso el algoritmo de clasificación tomó cinco propiedades para hacer la modelación, lo que permite evidenciar que este índice es el que presenta una mayor solidez teórica ya que es bien sabido que las propiedades que tienen mayor influencia sobre la sorción de metales pesados son el pH (Bradl, 2004; Ramos-Gómez et al., 2012; Tahervand y Jalali, 2016), la materia orgánica y las arcillas (Bradl, 2004; González-Flores et al., 2009).

En la disolución y sorción de metales pesados los 


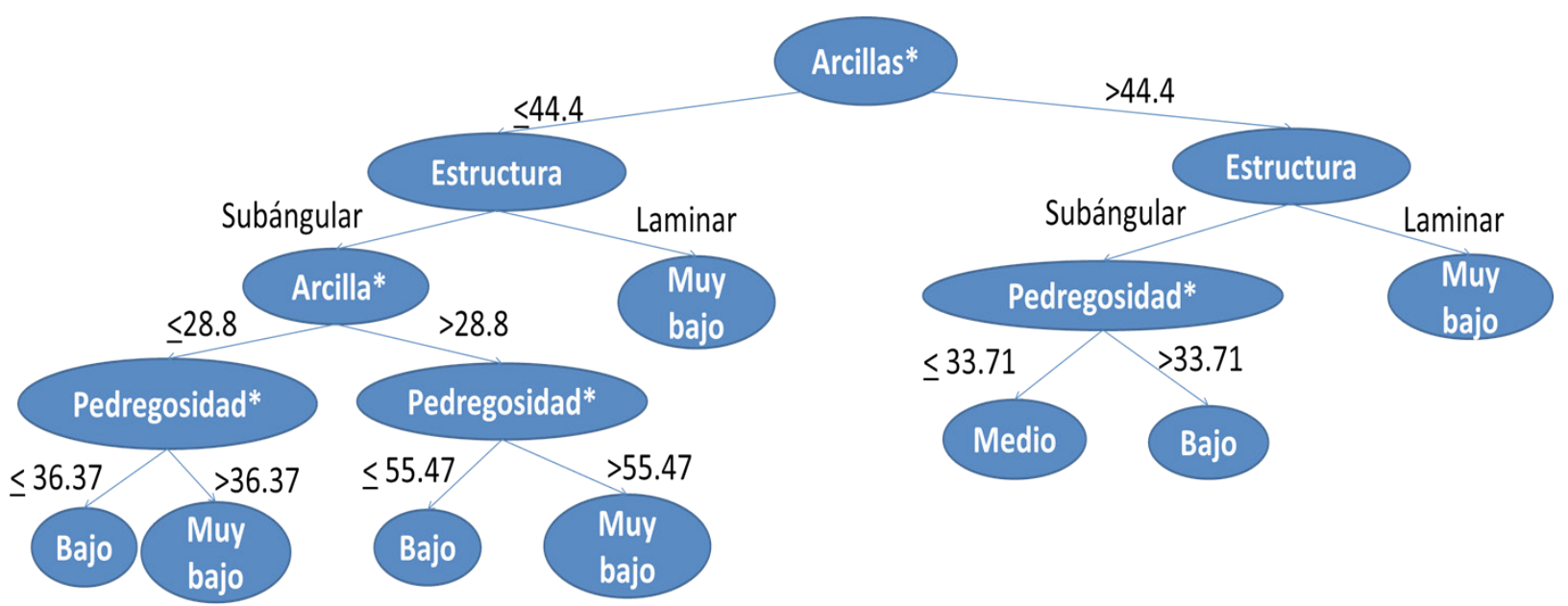

Figura 3 Árbol de decisión para clasificación del índice Lehmann.

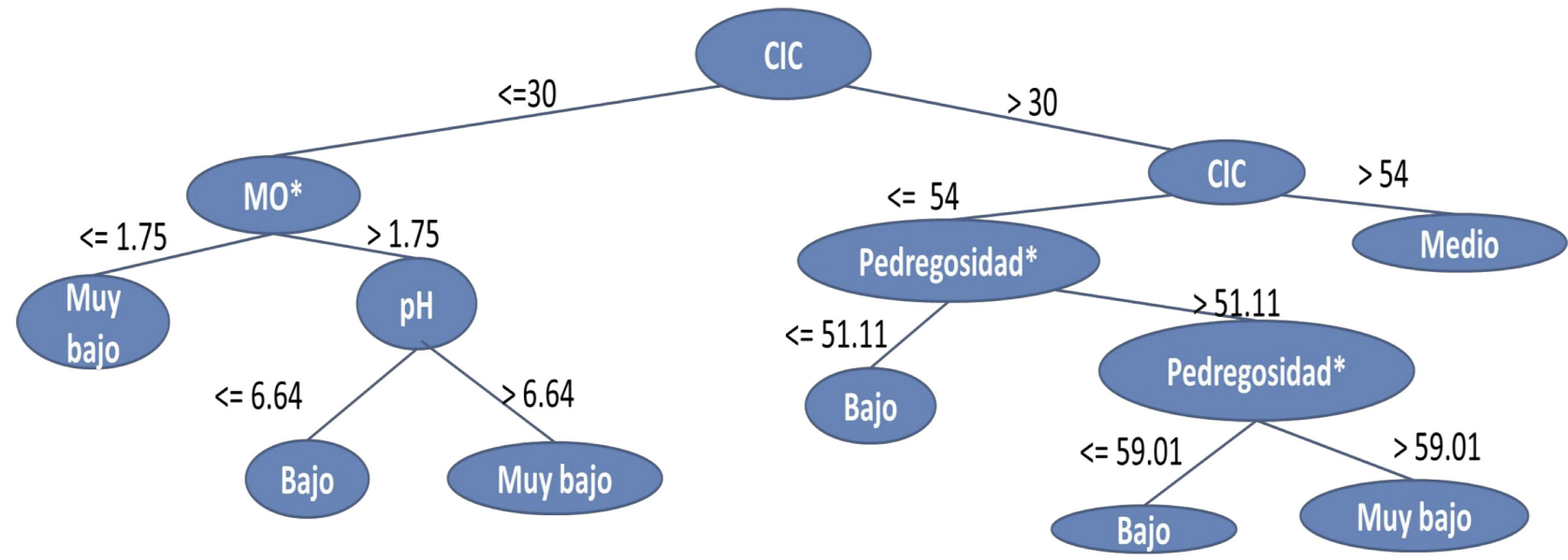

Figura 4 Árbol de decisión para clasificación del índice Lehmann modificado.

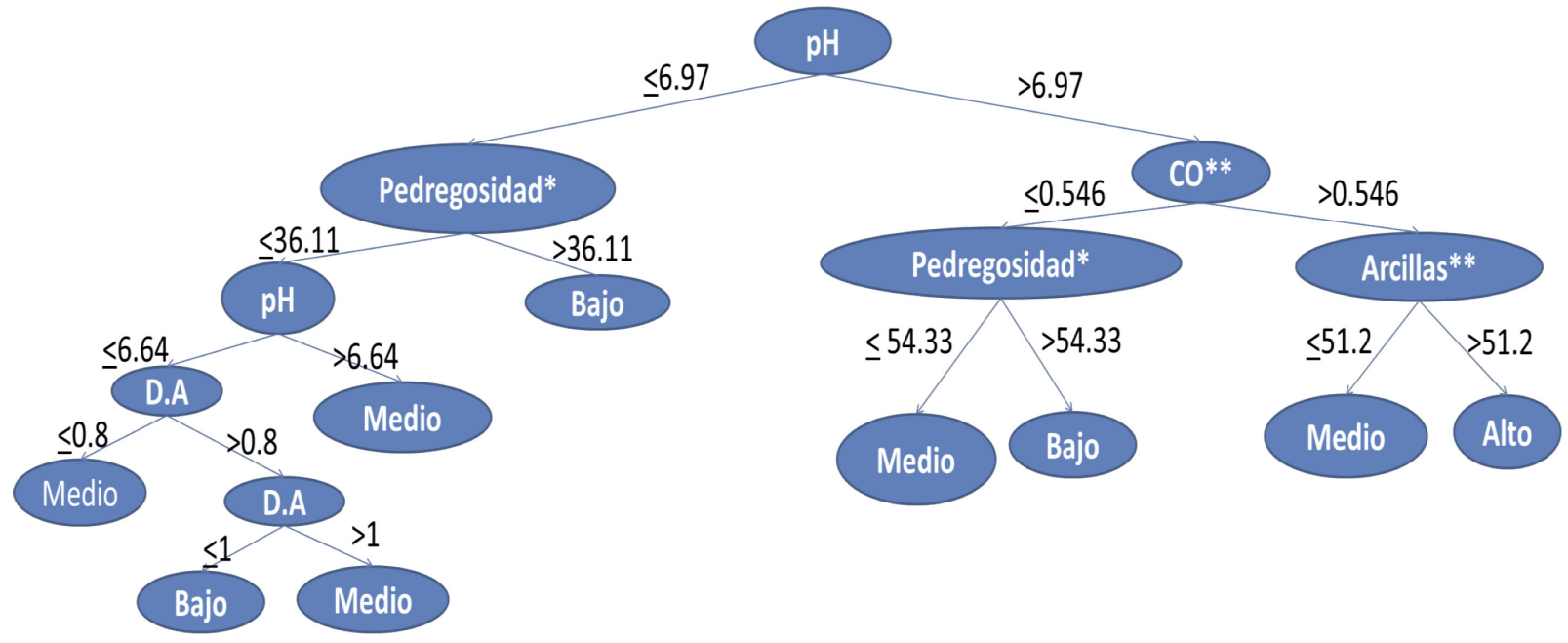

Figura 5 Árbol de decisión para clasificación del índice Bautista-Campuzano. 
factores más importantes son: el $\mathrm{pH}$ debido a las reacciones de hidrólisis de los cationes (Bradl, 2004; Ramos-Gómez et al., 2012; Tahervand y Jalali, 2016); el contenido de materia orgánica, debido a que los ácidos húmicos y fúlvicos contienen grupos funcionales como el carboxilo e hidroxilo (Mirsal, 2008); y las arcillas (Bradl, 2004; Ramos-Gómez et al., 2012). El árbol de decisión del índice "Bautista-Campuzano" modeló la clasificación de la sorción de MP conforme a la teoría de sorción del suelo anteriormente mencionada, ya que considera el $\mathrm{pH}$ en primera instancia (Bradl, 2004; Ramos-Gómez et al., 2012; Tahervand y Jalali, 2016) y posteriormente al carbono orgánico (Lair et al., 2007), seguido por arcillas, así como la pedregosidad y la densidad aparente, ambas relacionadas con la cantidad de tierra fina. De acuerdo con el coeficiente Kappa los tres índices son aceptables, ya que sus valores fueron mayores a 0.75 (Legrand y Nicoloyannis, 2005); sin embargo, la mayor solidez teórica la obtuvo el índice de sorción Bautista-Campuzano.

Tabla 6. Clases de los índices de contaminación por metales pesados y el área que cubren dentro de la Ciudad de Morelia, Michoacán.

\begin{tabular}{|l|c|c|}
\hline \multicolumn{1}{|c|}{ Clase } & Área (\%) & Área (ha) \\
\hline Muy baja & 3.85 & 489.6 \\
\hline Baja & 34.81 & 4426.5 \\
\hline Media & 44.26 & 5628.2 \\
\hline Alta & 17.08 & 2171.9 \\
\hline
\end{tabular}

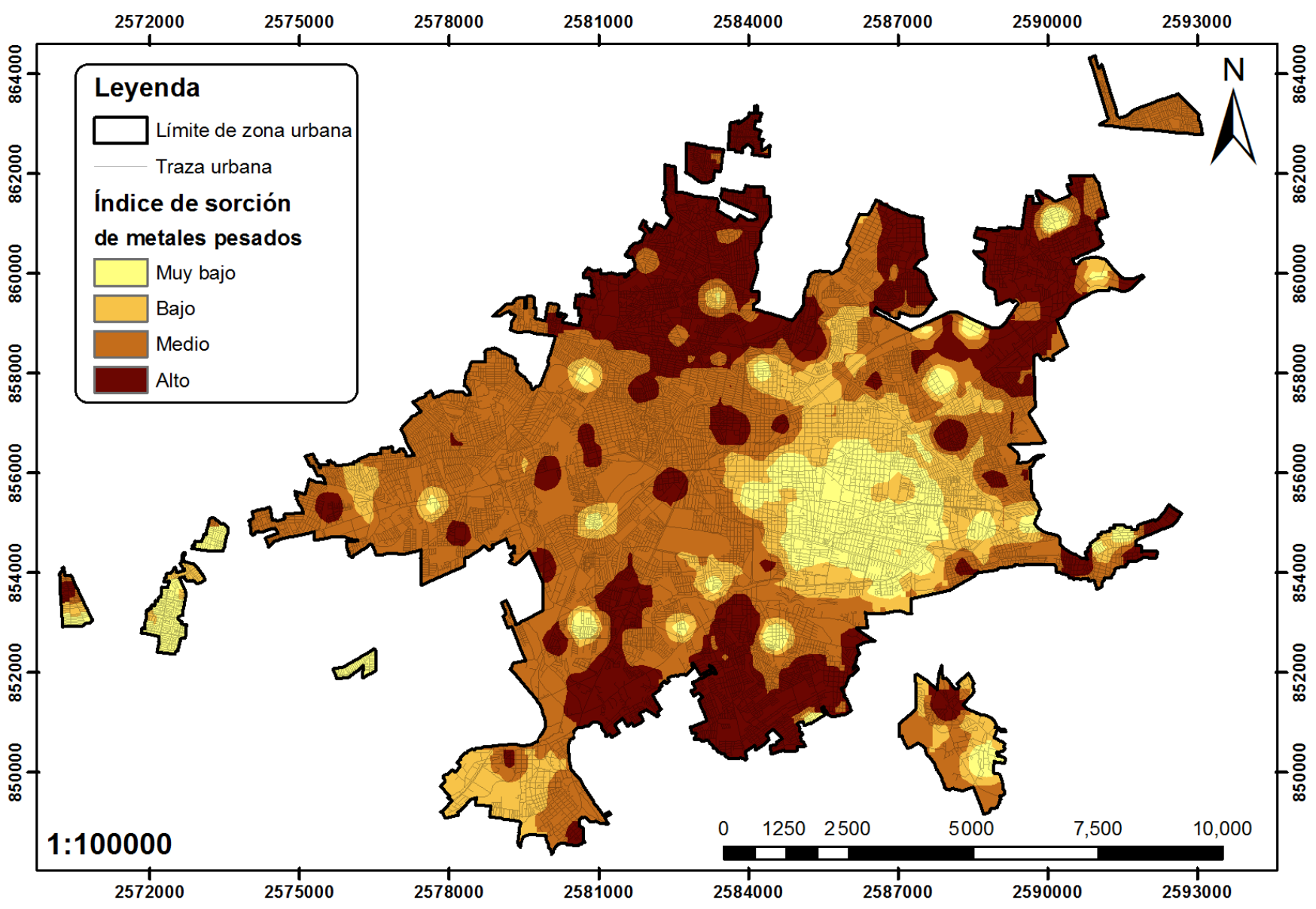

Figura 6 Mapa del índice de sorción de metales pesados en Morelia, Michoacán. 


\subsection{ANÁLISIS ESPACIAL}

Las clases de sorción de metales pesados de muy baja y baja ocupan un total de $38.66 \%$ de la ciudad de Morelia (Tabla 6, Figura 6). Desafortunadamente, esta superficie se corresponde con las áreas más pobladas al centro de la ciudad. Por el contrario, las clases de alta sorción se localizan en las áreas periurbanas donde la densidad de población es menor. Con esta información se pueden identificar las áreas que deben mejorar sus suelos. La adición de composta puede mejorar la estructura, aumentar el valor de $\mathrm{pH}$ y aumentar la CIC. Otra opción de mejoramiento de los suelos podría ser la incorporación de arcillas y carbonatos de calcio. Para futuras investigaciones se propone tomar en cuenta la carga contaminante y el tipo de uso de suelo (Bautista, 1999; Yao et al., 2012; Tahervand y Jalali, 2016).

Cabe resaltar que para otras ciudades es necesario realizar estudios de suelos que permitan determinar sus propiedades físicas y químicas, tanto de minerales que pueden precipitar los metales pesados, tales como los carbonatos, fosfatos, y sulfatos; como de los minerales que puedan adsorberlos, es el caso de los óxidos e hidróxidos de hierro, aluminio y manganeso y los alumino silicatos (Alumaa et al., 2001; Bradl, 2004; Lair et al., 2007; Sangiumsak y Punrattanasin, 2014).

En caso de que se tenga en cuenta un elemento contaminante se deberán seleccionar sólo aquellas propiedades de los suelos particularmente relacionadas con la sorción del elemento (Bradl, 2004; Tahervand y Jalali, 2016).

\section{Conclusiones}

Los tres índices aplicados a las propiedades de los suelos urbanos de la ciudad de Morelia mostraron congruencia entre las propiedades del suelo y las clases de sorción de metales pesados. Pero, el índice Bautista-Campuzano fue el que mostró mayor congruencia teórica según el árbol de de- cisión para clasificación, por ello se proponer utilizar dicho índice en los planes de manejo de los suelos urbanos.

La zona más poblada de la Ciudad de Morelia, en el estado de Michoacán, fue la que presentó la clase de baja capacidad de sorción de metales pesados, por lo que se considera urgente tomar decisiones y realizar acciones para proteger a la población.

\section{Agradecimientos}

Al CONACyT por el financiamiento al proyecto 169915. Carmen Delgado agradece al Consejo Nacional de Ciencia y Tecnología por la beca posdoctoral. A Hilda Rivas por su apoyo técnico en el laboratorio. A Alma Barajas, José Luis Cortés, Gabriela García, Daniel Baca y Luis D. Olivares por el apoyo técnico. A los árbitros por sus atinadas sugerencias.

\section{Fe de erratas}

Una versión de este manuscrito fue publicada en línea antes del cierre oficial de la edición de este número con las palabras "adsorción" y "adsorption" en lugar de "sorción" y "sorption". Se optó por la terminología existente, a pesar de que la palabra "sorción" no existe oficialmente en la lengua española (ver diccionario de la RAE), por el hecho que dicho término agrupa a los de "adsorción" y "absorption", es decir, pertenece a una categoría terminológica superior. En cambio, "sorption" sí existe en la lengua inglesa. Se tomó la decisión para evitar la confusión, en este trabajo, entre los efectos debidos a adsorción y los debidos a absorción.

\section{Referencias}

Aggarwal, C.C., 2015, Data mining: the textbook: Switzerland, Springer, 734 p. 
Aguilar-Reyes, B., Cejudo-Ruíz, R., MartínezCruz, J., Bautista, F., Goguitchaichvili, A., Carvallo, C., Morales, J., 2012, Ficus benjamina leaves as indicator of atmospheric pollution: A reconnaissance study: Studia Geophysica et Geodaetica, 56, 879-887.

Aguilar-Reyes, B., Bautista, F., Goguichaishvili, A., Quintana, P., Carvallo, C., Battu, J., 2013a, Rock-Magnetic properties of topsoils and urban dust from Morelia, México: implications for anthropogenic pollution monitoring in medium-size cities: Geofísica Internacional, 52, 12-33.

Aguilar-Reyes, B., Mejía, V., Goguichaishvili, A., Escobar, J., Bayona, G., Bautista, F., Morales, J., Ihl, T., 2013b, Reconnaissance environmental magnetic study of urban soils, dust and leaves from Bogotá, Colombia: Studia Geophysica et Geodaetica, 57, 74-54.

Alloway, B.J., Trevors, J.T., 2013, Heavy metals in soils: Trace metals and metalloids in soils and their bioavailability: Dordrecht, Springer, $613 \mathrm{p}$.

Alumaa, P., Steinnes, E., Kirso, U., Petersell, V., 2001, Heavy metal sorption by different Estonian soil types at low equilibrium solution concentrations: Proceedings of Estonian Academy of Sciences, 1(50), 104-15.

Areygue-Rocha, E., Garduño, V.H., Canuti, P., Casaglie, N., Iotti, A., Chiesa, S., 2002, Análisis geomecánico de la inestabilidad del escarpe La Paloma, en la Ciudad de Morelia, Michoacán, México: Revista Mexicana de Ciencias Geológicas, 19(2), 9-06.

Bautista, F., 1999, Introducción al estudio de la contaminación del suelo por metales pesados: Yucatán, México, Universidad Autónoma de Yucatán, 109 p.

Bautista, F., Cram Heydrich, S., Sommer Cervantes, I., 2011, Suelos, en Bautista, F., Palacio, J.L., Delfin, H., Paéz, R., Carmona, E., Delgado, C. (eds.), Técnicas de muestreo para manejadores de recursos naturales: Cd. de México, UNAM, CIGA, IG, 227-258.
Bautista, F., Gallegos-Tavera, M.A., Álvarez Arriaga, O., 2015, La evaluación automatizada de las funciones ambientales del suelo con base en datos de perfiles: Morelia, Michoacán, México, CIGA, UNAM, 109 p.

Bautista, F., García, E., Gallegos, A., 2016, The App SOC + a tool to estimate or/and calculate organic carbon in the soil profile: Journal of Applied Research and Technology, 14, 135-139.

Bradl, H.B., 2004, Adsorption of heavy metal ions on soils and soils constituents: Journal of Colloid and Interface Science, 277, 1-18.

Bronick, C., Lal, R., 2005, Soil structure and management: A review: Geoderma, 124, 3-22.

Cejudo-Ruíz, R., Bautista, F., Quintana, P., Delgado-Carranza, M.C., Aguilar, D., Goguichaishvili, A., Morales-Contreras, J.J., 2015, Correlación entre los elementos potencialmente tóxicos y propiedades magnéticas en suelos de la Ciudad de México para la determinación de sitios contaminados: definición de umbrales magnéticos: Revista Mexicana de Ciencias Geológicas, 32, 50-61.

Cerda, L.J., Villarroel del, P.L., 2008, Evaluación de la concordancia inter-observador en investigación pediátrica: Coeficiente de kappa: Revista Chilena de Pediatría, 79, 54-58.

Cortés, J.L., Bautista, F., Quintana, P., Aguilar, D., Gogichaishvili, A., 2015, El color del polvo urbano como indicador de contaminación por elementos potencialmente tóxicos: $\mathrm{El}$ caso de Ensenada, Baja California: Revista Chapingo Serie Ciencias Forestales y del Ambiente, 20, 255-266.

Davis, A.P., 2000, Chemical and Engineering aspects of heavy metals contaminated soils, Revista Internacional de Contaminación Ambiental, 16(4), 169-174.

Delgado, C., Pacheco, J., Cabrera, A., Batllori, E., Orellana, R., Bautista, F., 2010, Quality 
of groundwater for irrigation in tropical karst environment: The case of Yucatan, Mexico: Agriculture Water Management, 97, 1423-1433.

Delgado, C., Israde, I., Bautista, F., Gogichaishvili, A., Márquez, C., Gejudo, R., Morales, J., González, I., 2015, Metales pesados en suelos urbanos de Morelia Michoacán: influencia del uso del suelo y del tipo de vialidad: Ciencia Nicolaita, 65, 120-138.

Environmental Systems Research Institute (ESRI), 2004, An overview of the spatial statistics toolbox. ArcGIS 10.2 online help system (ArcGIS 10.2 Desktop), Redlands, CA, USA. Food and Agriculture Organization of the United Nations (FAO), 2006, Guidelines for soil description: Rome, $111 \mathrm{p}$.

González-Flores, E., Tornero-Campante, M.A., Ángeles-Cruz, Y., Bonilla y Fernández, N., 2009, Concentración total y especiación de metales pesados en biosólidos de origen urbano: Revista Internacional de Contaminación Ambiental, 25, 15-22.

Hall, M., Frank, E., Holmes, G., Pfahringer, B., Reutemann, P., Witten, I.H., 2009, The WEKA Data Mining Software: An Update: SIGKDD Explorations, 11, 10-18.

Hernández-Stefanoni, J.L., Ponce-Hernández, R., 2006, Mapping the spatial variability of plant diversity in a tropical forest: comparison of spatial interpolation methods: Environmental Monitoring and Assessment, 117, 307-334.

Ihl, T., Bautista, F., Cejudo-Ruíz, F.R.,Delgado, M.C., Quintana-Owen, P., Goguitchaichvili, A., 2015, Concentration of toxic elements in topsoils of Metropolitan area of Mexico City: A spatial analysis using Ordinary Kriging and Indicator Kriging: Revista Internacional de ContaminaciónAmbiental, 31, 42-62.

Instituto Nacional de Ecología y Cambio Climático, Secretaria Nacional del Medio Ambiente y Recursos Naturales (INECGSEMARNAT), 2010, Estudio de emisiones y actividad vehicular en Morelia, Michoacán, reporte final, $93 \mathrm{p}$.
Instituto Nacional de Estadística, Geografía e Informática (INEGI), 2010, Censos y conteos de población y vivienda (en línea), México, disponible en http://www.inegi. org.mx/lib/olap/consulta/general_ver4/ MDXQueryDatos.asp? \#Regreso\&c $=27770$, consultado 29 de Julio de 2016.

Instituto Nacional de Estadística, Geografía e Informática (INEGI), 2014, Automóviles registrados en circulación en la ciudad de Morelia Michoacán (en línea), México, publicado en Enero de 2014, disponible en http://www3.inegi.org.mx/sistemas/ biinegi/, consultado 29 de Julio de 2016.

Jordán, A., 2006, Manual de Edafología, España, Universidad de Sevilla, 144 p.

Lair, G.J., Gerzabeck, M.H., Haberhauer, G., 2007, Sorption of heavy metal on organic and inorganic soil constituents: Environmental Chemistry Letters, 5, 23-27.

Legrand, G., Nicoloyannis, N., 2005, Data preprocessing and kappa coefficient (abstract), en 10th International Conference: Regina, Canada, Slezak Dominik, Wang Guoyin, Szczuka Marcin, Düntsch Ivo y Yao Yiyu, 176-184.

Lehmann, A., Stahr, K., 2007, Nature and significance of anthropogenic urban soils: Soils Sediments, 7(4), 247-260.

Lehmann, A., David, S., Stahr, K., 2013, Technique for soil evaluation and categorization for natural and anthropogenic soils: bilingual-edition: Stuttgart, Hohenheimer Bodenkundliche Hefte, 232 p.

Liu, D., Li, Y., Ma, J., Li, C., Chen, X., 2016, Heavy metal pollution in urban soil from 1994 to 2012 in Kaifeng City, China: Water, Air and Soil Pollution, 227, 154 p.

Lu, S.G., Bai, Q., 2010, Contamination and potential mobility assessment of heavy metals in urban soils of Hangzhou, China: relationship with different land uses: Environmental Earth Science, 60, 148-490.

Mirsal, I.A., 2008, Soil Pollution: Origin, monitoring and remediation: Springer, 
Berlin, 310 p.

Nelson, D.W., Sommers, L.E., 1982, Total carbon, organic carbon and organic matter, en Page, A.L., Miller, R.H., Keeney, D.R. (eds.), Methods of soil analysis, Part. 2, Chemical and microbiological properties, Wisconsin, EUA, American Society of Agronomy and Soil Science Society of America, 539-577.

Okalebo, R., Gathua, K.W., Woomer,P.L., 1993, Laboratory methods of soil and plant analysis: A working manual. Tropical Soil Biology and Fertility Program TSBF, Kenya.88 pp.

Porta, J. López-Acevedo, M., Roquero, C., 2003, Edafología: para la agricultura y el medio ambiente: Silsoe, Mundi-Prensa, 929 p.

Ramos-Gómez, M., Avelar, J., Medel-Reyes, A., Yamamoto, L., Godínez, L., Ramírez, M., Guerra, R., Rodríguez, F., 2012, Movilidad de metales en sales procedentes del Distrito Minero de Guanajuato, México: Revista Internacional Contaminación Ambiental, 28(1), 49-59.

Rhoades, J., 1982, Cation Exchange Capacity, en Page, A.L. (ed.), Methods of Soil Analysis. Part 2. Agronomy Monograph N ${ }^{\circ}$ 9, Madison, WI, ASA \& SSSA, 178-190.

Robertson, G.P., 2008, GS+: Geostatistics for the Environmental Sciences. Gamma Design Software, Plainwell, Michigan, USA, 172 p.
Sangiumsak, N., Punrattanasin, P., 2014, Adsorption behavior of heavy metals on various soils: Polish Journal of Environmental Studies, 23(3),853-865.

Sánchez-Duque, A., Bautista, F., Gogichaishvili, A., Cejudo-Ruiz, R., Reyes-López, J., SolísDomínguez, F., Morales-Contreras, J., 2015, Evaluación de la contaminación ambiental a partir del aumento magnético en polvos urbanos. Caso de estudio para la ciudad de Mexicali, México: Revista Mexicana de Ciencias Geológicas, 32, 50-13.

Tahervand, S., Jalali M., 2016, Sorption, desorption, and speciation of $\mathrm{Cd}, \mathrm{Ni}$, and Fe by four calcareous soils as affected by $\mathrm{pH}$ : Environmental Monitoring and Assessment, $188,322 \mathrm{p}$.

Towhid, O.K., 2013, Soils: Principles, properties and management: Dordrecht,Springer, 286 p.

Yao, Z., Li, J., Xie, H., Yu, C., 2012, Review on remediation technologies of soil contaminated by heavy metals: Procedia Environmental Science, 16, 722-729.

Zhang, L., Ren, R., Chen, S., Dong, P., 2014, Spatial variability of surface sediment basis on geostatistical analysis in the littoral area of Yellow River delta, China: Indian Journal of Geo-Marine Sciences, 34, 463-472. 